\title{
Discovery of very-high-energy $\gamma$-ray emission from the vicinity of PSR J1913+1011 with HESS
}

\author{
F. Aharonian ${ }^{1,13}$, A. G. Akhperjanian ${ }^{2}$, U. Barres de Almeida ${ }^{8, \star}$, A. R. Bazer-Bachi ${ }^{3}$, B. Behera ${ }^{14}$, M. Beilicke ${ }^{4}$
} W. Benbow ${ }^{1}$, K. Bernlöhr ${ }^{1,5}$, C. Boisson ${ }^{6}$, O. Bolz ${ }^{1}$, V. Borrel ${ }^{3}$, I. Braun ${ }^{1}$, E. Brion ${ }^{7}$, A. M. Brown ${ }^{8}$, R. Bühler ${ }^{1}$, T. Bulik ${ }^{24}$, I. Büsching 9 , T. Boutelier ${ }^{17}$, S. Carrigan ${ }^{1}$, P. M. Chadwick ${ }^{8}$, L.-M. Chounet ${ }^{10}$, A. C. Clapson ${ }^{1}$, G. Coignet $^{11}$, R. Cornils ${ }^{4}$, L. Costamante ${ }^{1,28}$, M. Dalton ${ }^{5}$, B. Degrange ${ }^{10}$, H. J. Dickinson ${ }^{8}$, A. Djannati-Atai ${ }^{12}$, W. Domainko ${ }^{1}$, L. O'C. Drury ${ }^{13}$, F. Dubois ${ }^{11}$, G. Dubus ${ }^{17}$, J. Dyks ${ }^{24}$, K. Egberts ${ }^{1}$, D. Emmanoulopoulos ${ }^{14}$, P. Espigat ${ }^{12}$, C. Farnier $^{15}$, F. Feinstein ${ }^{15}$, A. Fiasson ${ }^{15}$, A. Förster ${ }^{1}$, G. Fontaine ${ }^{10}$, Seb. Funk ${ }^{5}$, M. Füßling ${ }^{5}$, Y. A. Gallant ${ }^{15}$, B. Giebels ${ }^{10}$, J. F. Glicenstein ${ }^{7}$, B. Glück ${ }^{16}$, P. Goret ${ }^{7}$, C. Hadjichristidis ${ }^{8}$, D. Hauser ${ }^{1}$, M. Hauser ${ }^{14}$, G. Heinzelmann ${ }^{4}$, G. Henri ${ }^{17}$, G. Hermann ${ }^{1}$, J. A. Hinton ${ }^{25}$, A. Hoffmann ${ }^{18}$, W. Hofmann ${ }^{1}$, M. Holleran ${ }^{9}$, S. Hoppe ${ }^{1}$, D. Horns ${ }^{18}$, A. Jacholkowska ${ }^{15}$, O. C. de Jager ${ }^{9}$, I. Jung ${ }^{16}$, K. Katarzyński ${ }^{27}$, E. Kendziorra ${ }^{18}$, M. Kerschhaggl ${ }^{5}$, B. Khélifi ${ }^{10}$, D. Keogh ${ }^{8}$, Nu. Komin ${ }^{15}$, K. Kosack ${ }^{1}$, G. Lamanna ${ }^{11}$, I. J. Latham ${ }^{8}$, A. Lemière ${ }^{12}$, M. Lemoine-Goumard ${ }^{10}$, J.-P. Lenain ${ }^{6}$, T. Lohse ${ }^{5}$, J. M. Martin ${ }^{6}$, O. Martineau-Huynh ${ }^{19}$, A. Marcowith ${ }^{15}$, C. Masterson ${ }^{13}$, D. Maurin ${ }^{19}$, G. Maurin ${ }^{12}$, T. J. L. McComb ${ }^{8}$, R. Moderski ${ }^{24}$, E. Moulin ${ }^{7}$, M. de Naurois ${ }^{19}$, D. Nedbal ${ }^{20}$, S. J. Nolan ${ }^{8}$, S. Ohm ${ }^{1}$, J.-P. Olive ${ }^{3}$, E. de Oña Wilhelmi ${ }^{12}$, K. J. Orford ${ }^{8}$, J. L. Osborne ${ }^{8}$, M. Ostrowski ${ }^{23}$, M. Panter ${ }^{1}$, G. Pedaletti ${ }^{14}$, G. Pelletier ${ }^{17}$, P.-O. Petrucci ${ }^{17}$, S. Pita $^{12}$, G. Pühlhofer ${ }^{14}$, M. Punch ${ }^{12}$, B. C. Raubenheimer ${ }^{9}$, M. Raue ${ }^{4}$, S. M. Rayner ${ }^{8}$, O. Reimer ${ }^{\star \star}$, M. Renaud ${ }^{1}$, J. Ripken ${ }^{4}$, L. Rob ${ }^{20}$, L. Rolland ${ }^{7}$, S. Rosier-Lees ${ }^{11}$, G. Rowell ${ }^{26}$, B. Rudak ${ }^{24}$, J. Ruppel ${ }^{21}$, V. Sahakian ${ }^{2}$, A. Santangelo ${ }^{18}$, R. Schlickeiser ${ }^{21}$, F. Schöck ${ }^{16}$, R. Schröder ${ }^{21}$, U. Schwanke ${ }^{5}$, S. Schwarzburg ${ }^{18}$, S. Schwemmer ${ }^{14}$, A. Shalchi ${ }^{21}$, H. Sol ${ }^{6}$, D. Spangler ${ }^{8}$, Ł. Stawarz ${ }^{23}$, R. Steenkamp ${ }^{22}$, C. Stegmann ${ }^{16}$, G. Superina ${ }^{10}$, P. H. Tam ${ }^{14}$, J.-P. Tavernet ${ }^{19}$, R. Terrier ${ }^{12}$, C. van Eldik ${ }^{1}$, G. Vasileiadis ${ }^{15}$, C. Venter ${ }^{9}$, J. P. Vialle ${ }^{11}$, P. Vincent ${ }^{19}$, M. Vivier ${ }^{7}$, H. J. Völk ${ }^{1}$, F. Volpe ${ }^{10}$, S. J. Wagner ${ }^{14}$, M. Ward ${ }^{8}$, A. A. Zdziarski ${ }^{24}$, and A. Zech ${ }^{6}$

(Affiliations can be found after the references)

Received 21 September 2007 / Accepted 20 February 2008

\section{ABSTRACT}

The HESS experiment, an array of four Imaging Atmospheric Cherenkov Telescopes with high sensitivity and large field-of-view, has been used to search for emitters of very-high-energy (VHE, $>100 \mathrm{GeV}$ ) $\gamma$-rays along the Galactic plane, covering the region $30^{\circ}<l<60^{\circ}, 280^{\circ}<l<330^{\circ}$, and $-3^{\circ}<b<3^{\circ}$. In this continuation of the HESS Galactic Plane Scan, a new extended VHE $\gamma$-ray source was discovered at $\alpha_{2000}=19^{\mathrm{h}} 12^{\mathrm{m}} 49^{\mathrm{s}}$, $\delta_{2000}=+10^{\circ} 09^{\prime} 06^{\prime \prime}$ (HESS J1912+101). Its integral flux between $1-10 \mathrm{TeV}$ is $\sim 10 \%$ of the Crab Nebula flux in the same energy range. The measured energy spectrum can be described by a power law $\mathrm{d} N / \mathrm{d} E \sim E^{-\Gamma}$ with a photon index $\Gamma=2.7 \pm 0.2_{\text {stat }} \pm 0.3$ sys. HESS J1912 +101 is plausibly associated with the high spin-down luminosity pulsar PSR J1913+1011. We also discuss associations with an as yet unconfirmed SNR candidate proposed from low frequency radio observation and/or with molecular clouds found in ${ }^{13} \mathrm{CO}$ data.

Key words. ISM: supernova remnants - gamma rays: observations

\section{Introduction}

A significant fraction of the recently discovered sources of veryhigh-energy $\gamma$-rays (VHE; $E>100 \mathrm{GeV}$ ) within the Galactic plane are associated with pulsar wind nebulae (PWNs) of high spin-down luminosity pulsars, such as G 18.0-0.7 (Aharonian et al. 2006d) and MSH 15-52 (Aharonian et al. 2005b), or with expanding shells of supernova remnants (SNRs) such as RX J1713.7-3946 (Aharonian et al. 2007a) and RX J0852.0 4622 (Aharonian et al. 2007b). The observed VHE $\gamma$-ray emission from these objects provides direct evidence for the existence of particles accelerated to energies of at least $\sim 10^{14} \mathrm{eV}$ (Aharonian et al. 2007a). In the case of accelerated electrons

\footnotetext{
* Supported by CAPES Foundation, Ministry of Education of Brazil.

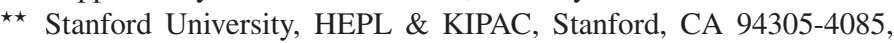
USA.
}

the VHE $\gamma$-rays are produced via inverse-Compton scattering on ambient photon fields, or, in case of dense environments, via non-thermal bremsstrahlung. In the case of accelerated hadrons, $\pi^{0}$ mesons and other hadrons are produced in interactions with nuclei in the interstellar medium. The $\gamma$-rays result from the decay of these particles, in particular of the $\pi^{0} \mathrm{~s}$.

Most of the Galactic objects expected to emit VHE $\gamma$-rays are related to some phase in the evolution of massive stars, either to their wind outflows or to PWNs and SNR shells formed after their collapse. Their spatial distribution is characterised by close clustering along the Galactic plane (rms in latitude $\sim 0.3^{\circ}$, Aharonian et al. 2006a). A systematic survey of the Galactic plane is therefore an effective approach to discovering new $\gamma$-ray sources and source classes. Due to its large field-of-view, its high sensitivity and its location in the southern hemisphere, the High Energy Stereoscopic System (HESS) is the VHE 

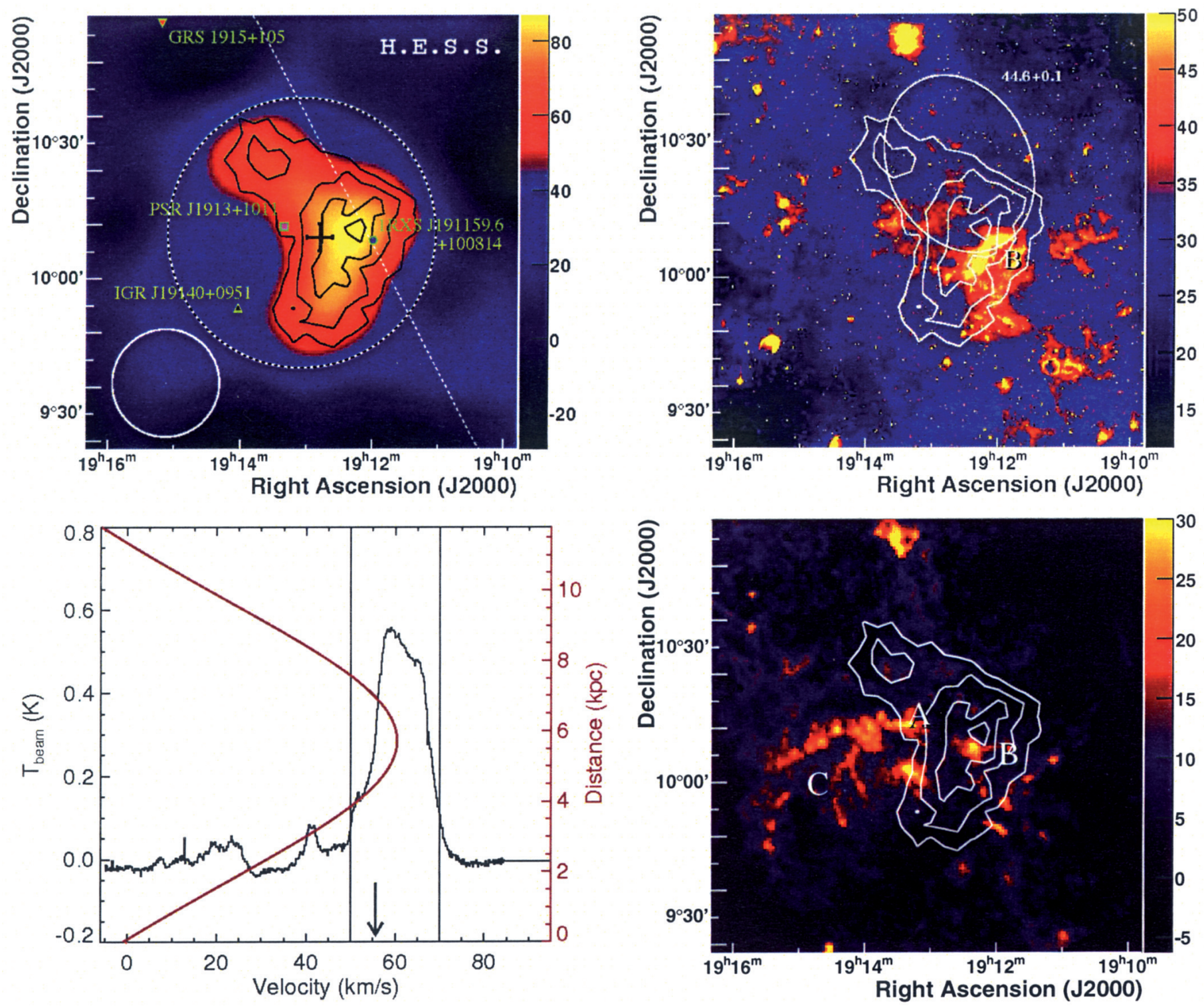

Fig. 1. Top, left: image of the VHE $\gamma$-ray excess from HESS J1912+101, smoothed with a Gaussian profile of $\sigma=0.13^{\circ}$ along each axis. Significance contours calculated for an integration radius of $\theta_{\text {cor }}=0.22^{\circ}$ are shown in black at 3.5, 4.5, 5.5 and 6.5 $\sigma$. The black cross indicates the best fit position of the source centroid together with its statistical errors. The dashed circle indicates the region from which the spectrum shown in Fig. 2 has been extracted. The positions of the pulsar PSR J1913+1011, the ROSAT source 1RXS J191159.6+100814, the INTEGRAL source IGR J19140+0951 and the microquasar GRS 1915+105 are marked. The Galactic plane is indicated by a white dotted line. The white solid circle illustrates the $68 \%$ containment radius of the Gaussian brightness profile used for smoothing convolved with the point spread function of the instrument. Top, right: image of the surface brightness (in units of $\mathrm{MJy} \mathrm{sr}^{-1}$ ) measured at $\sim 8.0 \mu \mathrm{m}$ within the Galactic Legacy Infrared Mid-Plane Survey Extraordinaire (GLIMPSE) using the Spitzer telescope (Benjamin et al. 2003). The white contours indicate the significance of the VHE $\gamma$-ray excess. The region marked as "B" is a complex of molecular clouds and HII-regions. The white ellipse illustrates the position and extension of the SNR candidate 44.6+0.1 proposed in the Clark Lake $30.9 \mathrm{MHz}$ Galactic plane survey (Kassim 1988). Bottom, left: velocity profile of ${ }^{13} \mathrm{CO}(J=1 \rightarrow 0)$ intensity at $110.2 \mathrm{GHz}$ from the Galactic Ring Survey (GRS, Jackson et al. 2006), integrated within the dashed circle shown in the top, left figure. The velocity resolution is $0.25 \mathrm{~km} \mathrm{~s}^{-1}$. The distance/velocity correspondence from the Galactic rotation model from Fich et al. (1989) is also shown. The velocity corresponding to the nominal distance of PSR J1913+1011 (4.48 kpc) (Manchester et al. 2005) is marked by an arrow together with the velocity range of the image shown in the bottom, right figure. Bottom, right: $\operatorname{GRS}{ }^{13} \mathrm{CO}(J=1 \rightarrow 0)$ intensity in units of $\mathrm{K} \mathrm{km} \mathrm{s}^{-1}$. Intensities are integrated in the velocity range $50-70 \mathrm{~km} \mathrm{~s}^{-1}$, corresponding to a distance of $\sim 5 \mathrm{kpc}$. The molecular cloud marked as "A" is coincident with PSR J1913+1011. In region "C" no significant VHE $\gamma$-ray excess was observed so far, even though it contains molecular clouds at a similar distance.

instrument currently best-suited to performing such a survey. The first phase of the survey has resulted in numerous detections of VHE $\gamma$-ray emitters in the inner Galactic region $|l|<30^{\circ}$ and $|b|<3^{\circ}$ (Aharonian et al. 2006a; Aharonian et al. 2005a). In 2005-2007 the survey has been extended, covering the regions $l=280^{\circ}-330^{\circ}$ and $l=30^{\circ}-60^{\circ}$. Here we present one of the new sources, HESS J1912+101, discovered during these observations, which is a good candidate for a VHE PWN such as HESS J1825-137 (Aharonian et al. 2006d), HESS J1718-385 and HESS J1809-193 (Aharonian et al. 2007c). 


\section{Observations and results}

The region of interest, which includes the microquasar GRS 1915+105 (Rodriguez et al. 1995), the Integral source IGR J19140+0951 (Hannikainen et al. 2004), the high spindown luminosity pulsar PSR J1913+1011 (Morris et al. 2002), as well as the SNR candidate 44.6+0.1 (Kassim 1988), was first targeted in 2004 as part of the observational programme on GRS 1915+105. In 2005, dedicated observations were taken on the supernova remnant W 49B (Harris et al. 1960); some of the pointings also cover the field-of-view of interest. In 2006, the region was observed again, as part of the extended HESS Galactic Plane Survey and with dedicated observations of IGR J19140+0951. PSR J1913+1011 was selected for dedicated observations as part of a systematic study of high spin-down luminosity pulsars, but was not observed due to the planned coverage of this region in other programmes. The combined data set was investigated using the standard survey analysis (a radius of the on-source region of $\theta_{\text {cut }}=0.22^{\circ}$, a ring background region of radius $0.8^{\circ}$ and hard cuts, which include a minimum requirement of 200 photo electrons per shower image, for $\gamma$-ray selection) as described in Aharonian et al. (2006a).

The analysis presented considers only observations pointed within $3^{\circ}$ of the original source candidate apparent within the Galactic plane survey analysis. The mean pointing offset is $1.1^{\circ}$. After quality selection to remove data affected by unstable weather conditions or hardware issues, the data set has an acceptance corrected live time (equivalent time spent at an offset of $0.5^{\circ}$ ) of $20.8 \mathrm{~h}$ at the centre of the emission. The zenith angles of the observations range from $33^{\circ}$ to $54^{\circ}$ leading to a typical energy threshold of $\sim 800 \mathrm{GeV}$. Figure 1 (top, left) shows the excess count map of the $1.6^{\circ} \times 1.6^{\circ}$ region around the source smoothed with a Gaussian profile of width $0.13^{\circ}$ to reduce statistical fluctuations. A clear excess of VHE $\gamma$-rays is observed with a peak statistical significance of $7.5 \sigma$ for an integration radius of $\theta_{\text {cut }}=0.22^{\circ}$. As the source was discovered as part of the HESS Galactic Plane Survey, the statistical trials associated with this survey must be taken into account (see Aharonian et al. 2006a). A very conservative estimate of the number of trials which accounts for the large number of sky positions probed $\left(\sim 5 \times 10^{5}\right)$ leads to a post-trials significance of $5.5 \sigma$. Fitting the uncorrelated excess count map with a symmetric Gaussian profile convolved with the point spread function of the instrument leads to a best fit position of $\alpha_{2000}=19^{\mathrm{h}} 12^{\mathrm{m}} 49^{\mathrm{s}}, \delta_{2000}=+10^{\circ} 09^{\prime} 6^{\prime \prime}$, with a $3^{\prime}$ statistical error in each coordinate, as indicated by the black cross in Fig. 1 (top, left). HESS J1912+101 is clearly extended with an intrinsic Gaussian width of $0.26^{\circ} \pm 0.03^{\circ}$ stat, with a fit probability for a Gaussian source profile of $9 \%$. Ellipsoidal fits do not improve the fit probability. No further statements about the morphology are possible at the level of statistics available at the moment.

For the spectral analysis only observations pointed within $2.0^{\circ}$ of the centre of the source were used, to remove events with large uncertainties in reconstructed energy. The live time of the remaining dataset is $18.7 \mathrm{~h}$. The spectrum was determined within a circular region of $0.5^{\circ}$ radius (indicated by a dashed circle in Fig. 1 (top, left)), which represents an $\sim 90 \%$ enclosure of the excess, chosen as a compromise between optimal signal to noise ratio and independence of source morphology. To minimise spectral uncertainties, the background was estimated from regions with equal offset from the centre of the field-of-view as described in Berge et al. (2007). The dataset contains a large fraction of observations targeted at other possible sources within the field-of-view (e.g. GRS 1915+105) and

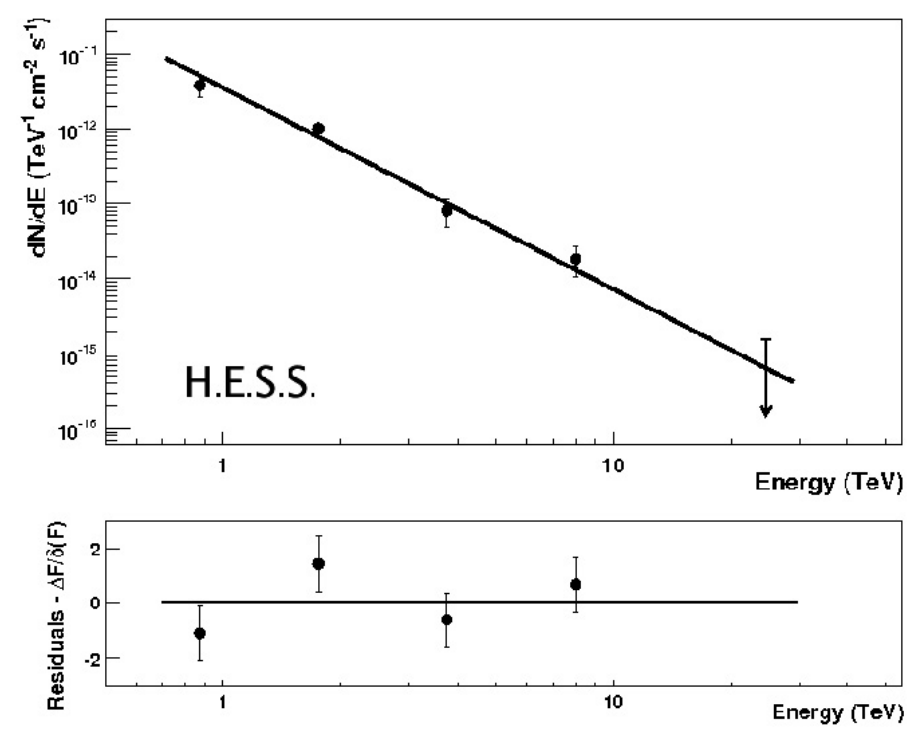

Fig. 2. Differential energy spectrum of HESS J1912+1011, extracted from the circular region indicated in Fig. 1 (top, left). Events with energies between 0.55 and $55 \mathrm{TeV}$ were used in the determination of the spectrum. They were binned with three bins per decade - the resulting number of bins is 6 - and then fitted with a power law (solid line). The two highest energy points of the spectrum were merged and are shown here as a $2 \sigma$ upper limit.

was taken over a time period of three years. Furthermore, due to the source extension, the observations of HESS J1912+101 have rather uneven exposure across the region and coverage over time. Consideration of only nearby HESS pointing positions, use of the reflected-region background model and the estimation of the time-dependent optical response of the system, corrects for these variations. However, these factors lead to somewhat increased systematic errors with respect to typical HESS results. Within the large integration circle 276 excess events were found, corresponding to a statistical significance of $7.5 \sigma$ (pre-trials) for this dataset used for spectral analysis. The spectrum shown in Fig. 2 can be described by a power law: $\mathrm{d} N / \mathrm{d} E=\Phi_{0}(E / 1 \mathrm{TeV})^{-\Gamma}$ with photon index $\Gamma=2.7 \pm 0.2_{\text {stat }} \pm 0.3_{\text {sys }}$ and flux normalisation $\Phi_{0}=\left(3.5 \pm 0.6_{\text {stat }} \pm 1.0_{\text {sys }}\right) \times 10^{-12} \mathrm{~cm}^{-2} \mathrm{~s}^{-1} \mathrm{TeV}^{-1}$. The fit has a $\chi^{2} / \mathrm{ndf}=6.3 / 4$. The integral flux between 1 and $10 \mathrm{TeV}$ is about $9 \%$ of the flux of the Crab Nebula in the same energy range (Aharonian et al. 2006c).

\section{Possible associations}

The celestial region around HESS J1912+101 hosts several potential counterparts. While a superposition of two or more is also possible, each of the following objects could individually account for the observed $\gamma$-ray emission:

The most plausible counterpart candidate, both in positional and energetic connection to HESS J1912+101 is the pulsar PSR J1913+1011 (Morris et al. 2002), which is slightly offset from the HESS source's best fit position by $\sim 0.15^{\circ}$, spatially consistent at the $\sim 3 \sigma$ level. It is a rather energetic pulsar with a spin-down luminosity of $2.9 \times 10^{36} \mathrm{erg} \mathrm{s}^{-1}$, a spindown age of $\tau_{\mathrm{c}} \approx 1.7 \times 10^{5}$ years, a spin period of $36 \mathrm{~ms}$ and a distance estimated from dispersion measurements of $4.48 \mathrm{kpc}$ (Manchester et al. 2005). The pulsar is sufficiently energetic to power the HESS source with an implied conversion efficiency from rotational energy to $1-10 \mathrm{TeV} \gamma$-rays of $\epsilon=0.5 \%$, comparable to the efficiency inferred for other VHE PWN candidates such as HESS J1718-385 and HESS J1809-193 
(Aharonian et al. 2007c). Its distance would result in a projected size of HESS J1912+101 of $\sim 70$ pc.

These characteristics suggest an association of the VHE emission with the pulsar's wind nebula, similar to other PWN associations such as Vela X (Aharonian et al. 2006b), HESS J1825-137 (Aharonian et al. 2006d) and MSH 15-52 (Aharonian et al. 2005b). Compared with these examples, HESS J1912+101 would be the oldest candidate for a VHE emitting PWN. In leptonic models of VHE $\gamma$-ray production, a PWN is expected to also emit non-thermal X-rays. Two Chandra observations within the region of interest are currently available. The first (Obs. Id 4590) was targeted on EXO 1912+097 (Lu et al. 1996), which is coincident with and possibly the same object as IGR J19140+0951 (Hannikainen et al. 2004). The overlap of this observation with HESS J1912+101 is marginal. The second (Obs. Id 3854) was targeted on the pulsar PSR J1913+1011 and covers a larger fraction of the HESS source. No X-ray PWN was reported at the position of PSR J1913+1011 (see Gotthelf et al. 2004). Existing Chandra observations do not allow for a counterpart search on the same spatial scale as the HESS source, and a detailed analysis of the Chandra data is beyond the scope of this paper. Despite considerably less sensitivity, the ASCA satellite with its larger field-of-view seems more suitable for counterpart studies on larger scales. Two ASCA observations coincide with HESS J1912+101. The first (Obs. Id 57005060) covers the whole HESS source but suffers strongly from stray light contamination, the second (Obs. Id 57005070) barely covers the peak of the VHE emission. Therefore no ASCA data has been analysed for this paper.

In the emerging picture of PWN associations the VHE emission peak is often found offset from the pulsar position (e.g. HESS J1825-137, MSH 15-52, HESS J1718-385 and HESS J1809-193). These offsets can be explained in terms of the proper motion of the pulsar and/or the expansion of the SNR/PWN into an inhomogeneous medium (see e.g. Blondin et al. 2001). In the case of HESS J1912+101 the observed offset could be explained by proper motion assuming a velocity of $\sim 60 \mathrm{~km} \mathrm{~s}^{-1}$ (to travel the $\sim 10 \mathrm{pc}$ from the centroid of the VHE emission to the current pulsar location in $1.7 \times 10^{5}$ years). The hypothesis that the PWN of PSR J1913+1011 (and the associated SNR) is expanding into an inhomogeneous medium seems quite plausible as the ${ }^{13} \mathrm{CO}(J=1 \rightarrow 0)$ emission line measurements taken at $110.2 \mathrm{GHz}$ indicate the existence of "clumpy" molecular material (in particular the area "A" as indicated in Fig. 1 (bottom, right)) at the pulsar position and roughly at its distance. Such a scenario for an asymmetric nebula was also proposed for HESS J1825-137 (Aharonian et al. 2006d). HESS J1912+101 and HESS J1825-137 share several similarities: 1) similar offset of the VHE emission region from the pulsar position; 2) similar angular size ( $\mathrm{rms}$ size of $0.24^{\circ}$ for HESS J1825-137 and $0.26^{\circ}$ for HESS J1912+101); 3) similar distances (inferred from dispersion measurements of the corresponding pulsars to be $\sim 4 \mathrm{kpc}$ ). The intrinsic sizes are hence both $\sim 40 \mathrm{pc}$. In a leptonic scenario, the cooling timescale, in combination with the propagation speed of the lowest energy (and hence longest-lived) electrons which upscattering soft photons to energies detectable by HESS, limit the extent of the emission region. As argued by Aharonian et al. (2006d) synchrotron cooling is likely to be the dominant energy-loss process for magnetic field strengths $\mathrm{B}>3 \mu \mathrm{G}$ and multi-TeV electrons. For scattering in the Thomson regime by cosmic microwave background radiation (CMBR) photons, the parent electron population responsible for the VHE $\gamma$-rays detected by HESS have energies $10-100 \mathrm{TeV}$. The magnetic field strength within the extended X-ray PWN of PSR B1823-13 suggested by Gaensler et al. (2003) is $\sim 10 \mu \mathrm{G}$. Considering the different sizes of the X-ray and VHE $\gamma$-ray emission regions $\left(5^{\prime}\right.$ versus $\left.60^{\prime}\right)$ for PSR B1823-13, the magnetic field strength in the outer VHE nebula should be lower than in the more compact X-ray nebula (see de Jager et al. 2008). Here we assume similar magnetic field strengths for HESS J1825-137 and HESS J1912+101. The corresponding lifetime due to synchrotron losses of electrons upscattering CMBR photons to energies $E_{\gamma}$ is given by

$\tau_{\text {synch }} \approx 3 \times 10^{4}\left(\frac{B}{5 \mu \mathrm{G}}\right)^{-2}\left(\frac{E_{\gamma}}{\mathrm{TeV}}\right)^{-1 / 2}$ years.

Even for a magnetic field strength of $5 \mu \mathrm{G}$ - a value similar to the interstellar magnetic field - and $1 \mathrm{TeV} \gamma$-rays is the synchrotron cooling time scale for the upscattering electrons a factor $\sim 6$ lower than the age of PSR J1913 $+1011\left(\tau_{\mathrm{c}}=1.7 \times 10^{5}\right.$ years $)$ and still close to the age of PSR B1823 $-13\left(\tau_{\mathrm{c}}=2.1 \times 10^{4}\right.$ years $)$. This provides a natural explanation for the similar sizes of HESS J1825-137 and HESS J1912+101 even though the spindown ages of the corresponding pulsars are different by a factor of $\sim 8$. Assuming the transport process to be dominantly advective rather than diffusive, the necessary average flow speed needed to drive electrons from the position of the pulsar to the edge of the $\gamma$-ray nebula $\left(D^{\text {trav }} \approx 40 \frac{d}{4.5 \mathrm{kpc}} \mathrm{pc}\right)$ within the synchrotron loss time is

$v=\frac{D^{\text {trav }}}{\tau_{\text {synch }}} \approx 1400\left(\frac{d}{4.5 \mathrm{kpc}}\right)\left(\frac{B}{5 \mu \mathrm{G}}\right)^{2}\left(\frac{E_{\gamma}}{\mathrm{TeV}}\right)^{1 / 2} \mathrm{~km} \mathrm{~s}^{-1}$.

This speed is fairly large, but may not be implausible if the associated SNR expanded at least in part into the low-density phase of the interstellar medium. Assuming an energy-independent advection speed, Eq. (2) predicts an energy-dependent morphology for the nebula, as was observed for HESS J1825-137, where the photon index changes with distance by $\Delta \Gamma \approx 0.6$ (Aharonian et al. 2006d). With the available statistics it is not possible to extract position-resolved spectra for HESS J1912+101 to test this hypothesis also in this case. We also note that a possibly significant fraction of the observed VHE emission could also result from IC scattering of higher energy target photons, e.g. emission from the close-by molecular clouds and HII-regions as visible in Fig. 1 (top, right). This component of the VHE emission would probe lower energy electrons with significantly higher cooling times. Its size would therefore be limited not by the cooling time but by the age of the system.

Whilst the spin-down powers of PSR B1823-13 and PSR J1913+1011 are very similar $\left(\sim 3 \times 10^{36} \mathrm{erg} \mathrm{s}^{-1}\right)$, HESS J1825-137 is a more luminous source than HESS J1912+101, resulting in fairly different apparent VHE conversion efficiencies of $3 \%$ for the former and $0.5 \%$ for the latter. The spectral indices of the VHE sources are, however, compatible within statistical uncertainties $\left(\Gamma=2.38 \pm 0.02_{\text {stat }} \pm 0.15_{\text {sys }}\right.$ and $\left.\Gamma=2.7 \pm 0.2_{\text {stat }} \pm 0.3_{\text {sys }}\right)$. Assuming a continous injection of electrons with a power law $\mathrm{d} N / \mathrm{d} E \approx E^{-\alpha}$ where $\alpha=2.0 \ldots 2.2, \alpha$ is increased to $3.0 \ldots 3.2$ by radiative cooling, and a VHE $\gamma$-ray spectrum with a photon index of 2.0 to 2.1 is expected above a synchrotron break energy of $\sim 30 \mathrm{GeV}$ for HESS J1912+101. In Aharonian et al. (2005c) different scenarios were discussed to explain the observed softer photon index, including the effect of Klein-Nishina corrections to the IC scattering cross-section and the superposition of IC spectra arising from electrons injected in the past. Both these arguments are equally applicable to HESS J1912+101. 
Another candidate object for the observed emission is the shell of a hypothetical supernova remnant which would be rather old if associated with the birth of PSR J1913+1011 (spin-down age $1.7 \times 10^{5}$ years). In this scenario, the observed $\gamma$-ray emission could be explained as the result of the interaction of accelerated particles from the SNR with dense molecular clouds. A candidate for a supernova remnant assocation is 44.6+0.1, an yet unconfirmed SNR candidate proposed in the Clark Lake $30.9 \mathrm{MHz}$ Galactic plane survey (Kassim 1988) and further investigated by Gorham et al. (1990). The estimated position and size of this object are illustrated by a white ellipse in Fig. 1 (top, right). 44.6+0.1 overlaps with the VHE emission region, but the overall match in morphology is rather poor. However, the resolution from the Clark Lake survey is insufficient for a firm conclusion about the morphology and only part of the remnant might be visible at $30.9 \mathrm{MHz}$. No SNR has been detected in X-rays. The only published keV X-ray sources in this region are the faint ROSAT sources 1RXS J191159.6+100814, 1RXS J191254.3+103807 and 1RXS J191114.8+102102 (Voges et al. 2000). Such a lack of X-ray emission could be explained using for example the model of Yamazaki et al. (2006), in which an age of the SNR of $1.7 \times 10^{5}$ years would imply a rather low $\mathrm{X}$-ray to $\mathrm{TeV}$ energy flux ratio of $\sim 0.01$ (for the energy ranges 2-10 $\mathrm{keV}$ and $1-10 \mathrm{TeV}$ ). In this scenario the spatial distribution of the VHE emission should then trace the distribution of the molecular clouds which act as target material. Such clouds have been observed in the vicinity of PSR J1913+1011 (indicated as area "B" in Fig. 1 (top, right)) in infrared at $\sim 8 \mu \mathrm{m}$ (Fig. 1 (top, right)), in the ${ }^{13} \mathrm{CO}$ measurements presented above (Fig. 1 (bottom, right)) and in the $\operatorname{GRS} \operatorname{CS}(J=2 \rightarrow 1)$ map of the region. The best distance estimate for $\mathrm{G} 44.3+0.1$, an HII region located within this region and coincident with 1RXS J191159.6+100814, is $3.9 \mathrm{kpc}$ (from HI absorption measurements, Kuchar et al. 1994), which - given the uncertainties of the two methods - would place it at a distance consistent with that of the pulsar ( $4.48 \mathrm{kpc}$, from dispersion measurements). The VHE emission peaks close to this dense region. There is, however, no evidence for an overall spatial correlation of the $\gamma$-ray emission with molecular clouds (see e.g. area "C" in Fig. 1 (bottom, right)). In summary, we find little evidence to support the scenario of an old SNR causing the observed VHE $\gamma$-ray signal, but can not exclude this hypothesis on the basis of the available experimental data.

Other celestial objects in the vicinity of HESS J1912+101 include the microquasar GRS $1915+105$ and the Integral source IGR J19140+0951. GRS $1915+105$ is $\sim 1^{\circ}$ away from the centroid of the VHE emission. The suggested distances to GRS 1915+105 (12.5 kpc, Rodriguez et al. 1995; and $6.5 \mathrm{kpc}$, Kaiser et al. 2005) would correspond to a projected distance to the centroid of HESS J1912+101 of 110 to 220 pc and the suggested jet termination sites (IRAS 19132+1035, IRAS 19124+1106) are both situated outside the VHE emission region. A possible association of GRS 1915+105 and HESS J1912+101 is therefore considered as unlikely. The relative position of IGR J19140+0951, which is probably a Super Giant X-ray binary (Nespoli et al. 2007), renders a dominant contribution to the VHE emission from this object unlikely as well.

\section{Summary}

The continuation of the HESS survey of the Galactic plane has resulted in the discovery of a new extended VHE $\gamma$-ray source, HESS J1912+101. The complexity of the vicinity of
HESSJ $1912+101$ at other wavebands does not allow us to make a firm conclusion on the nature of the source yet. The most plausible scenario appears to be that the observed emission is associated with the high spin-down luminosity pulsar PSR J1913+1011, which would make HESS J1912+101 a $\gamma$-ray PWN similar to HESS J1825-137 in both the characteristics of the observed $\gamma$-ray emission and the energetics of the associated pulsar. Ultimately, an energy-dependent morphology change needs to be established in further HESS observations to confirm the similarity with HESS J1825-137 as argued here. Also the PWN needs to be detected in deep X-ray observations. An alternative possibility is the interaction of accelerated particles from an old SNR with molecular material in a dense region in the interstellar medium. Neither of the two scenarios is fully supported by existing multiwavelength data, but the existence of an energetic pulsar within the VHE emission region favours an association with a PWN, although the confirmation of a PWN at other wavebands is currently pending.

Acknowledgements. The support of the Namibian authorities and of the University of Namibia in facilitating the construction and operation of HESS is gratefully acknowledged, as is the support by the German Ministry for Education and Research (BMBF), the Max Planck Society, the French Ministry for Research, the CNRS-IN2P3 and the Astroparticle Interdisciplinary Programme of the CNRS, the UK Science and Technology Facilities Council (STFC), the IPNP of the Charles University, the Polish Ministry of Science and Higher Education, the South African Department of Science and Technology and National Research Foundation, and by the University of Namibia. We appreciate the excellent work of the technical support staff in Berlin, Durham, Hamburg, Heidelberg, Palaiseau, Paris, Saclay, and in Namibia in the construction and operation of the equipment.

This publication makes use of molecular line data from the Boston UniversityFCRAO Galactic Ring Survey (GRS). The GRS is a joint project of Boston University and Five College Radio Astronomy Observatory, funded by the National Science Foundation under grants AST-9800334, AST-0098562, AST0100793, AST-0228993, \& AST-05076

\section{References}

Aharonian, F., Akhperjanian, A. G., Aye, K.-M., et al. (HESS Collaboration) 2005a, Science, 307, 1938

Aharonian, F., Akhperjanian, A. G., Aye, K.-M., et al. (HESS Collaboration) 2005b, A\&A, 435, L17

Aharonian, F., Akhperjanian, A. G., Bazer-Bachi, A. R., et al. (HESS Collaboration) $2005 \mathrm{c}$, A\&A, 442, L25

Aharonian, F., Akhperjanian, A. G., Bazer-Bachi, A. R., et al. (HESS Collaboration) 2006a, ApJ, 636, 777

Aharonian, F., Akhperjanian, A. G., Bazer-Bachi, A. R., et al. (HESS Collaboration), 2006b, A\&A, 448, L43

Aharonian, F., Akhperjanian, A. G., Bazer-Bachi, A. R., et al. (HESS Collaboration) 2006c, A\&A, 457, 899

Aharonian, F., Akhperjanian, A. G., Bazer-Bachi, A. R., et al. (HESS Collaboration) 2006d, A\&A, 460, 365

Aharonian, F., Akhperjanian, A. G., Bazer-Bachi, A. R., et al. (HESS Collaboration) 2007a, A\&A, 464, 235

Aharonian, F., Akhperjanian, A. G., Bazer-Bachi, A. R., et al. (HESS Collaboration) 2007b, ApJ, 661, 236

Aharonian, F., Akhperjanian, A. G., Bazer-Bachi, A. R., et al. (HESS Collaboration) 2007c, ArXiv e-prints, 705

Benjamin, R. A., Churchwell, E., Babler, B. L., et al. 2003, PASP, 115, 953

Berge, D., Funk, S., \& Hinton, J. 2007, A\&A, 466, 1219

Blondin, J. M., Chevalier, R. A., \& Frierson, D. M. 2001, ApJ, 563, 806

de Jager, O. 2008 Lect. Notes Phys., in press

Fich, M., Blitz, L., \& Starck, A. A. 1989, ApJ, 342, 272

Gaensler, B. M., Schulz, N. S., Kaspi, V. M., et al. 2003, ApJ, 588, 441

Gorham, P. W. 1990, ApJ, 364, 187

Gotthelf, E. V. 2004, IAU Symp., 218, 225

Hannikainen, D. C., Rodriguez, J., Cabanac, C., et al. 2004, A\&A, 423, L17

Harris, D. E., \& Roberts, J. A. 1960, PASP, 72, 237

Jackson, J. M., Rathborne, J. M., Shah, R. Y., et al. 2006, ApJS, 163, 145

Kaiser, C. R., Sokoloski, J. L., Gunn, K. F., et al. 2005, Ap\&SS, 300, 283

Kassim, N. E. 1988, ApJ, 328, L55

Kuchar, T. A., \& Bania, T. M. 1994, ApJ, 436, 117 
Lu, F. J., Li, T. P., Sun, X. J., et al. 1996, A\&AS, 115, 395

Manchester, R. N., Hobbs, G. B., Teoh, A., et al. 2005, AJ, 129, 1993

Morris, D. J., Hobbs, G., Lyne, A. G., et al. 2002, MNRAS, 335, 275

Nespoli, E., Fabregat, J., \& Mennickent, R. 2007, The Astronomer's Telegram, 983, 1

Rodriguez, L. F., Gerard, E. \& Mirabel, I. F. 1995, ApJS, 101, 173

Rodriguez, J., Cabanac, C., Hannikainen, D. C., et al. 2005, A\&A, 432, 235

Voges, W., Aschenbach, B., Boller, T., et al. 2000, VizieR Online Data Catalog, 9029

Yamazaki, R., Kohri, K., Bamba, A., et al. 2006, MNRAS, 371, 1975

\footnotetext{
${ }^{1}$ Max-Planck-Institut für Kernphysik, Heidelberg, Germany

e-mail: stefan.hoppe@mpi-hd.mpg.de

2 Yerevan Physics Institute, Armenia

3 Centre d'Étude Spatiale des Rayonnements, CNRS/UPS,

Toulouse, France

${ }^{4}$ Universität Hamburg, Institut für Experimentalphysik, Germany

5 Institut für Physik, Humboldt-Universität zu Berlin, Germany

${ }^{6}$ LUTH, Observatoire de Paris, CNRS, Université Paris VII, Meudon, France

7 DAPNIA/DSM/CEA, CE Saclay, Gif-sur-Yvette, France

8 University of Durham, Department of Physics, UK

9 Unit for Space Physics, North-West University, Potchefstroom, South Africa

${ }_{10}$ Laboratoire Leprince-Ringuet, Ecole Polytechnique, CNRS/IN2P3, Palaiseau, France

${ }^{11}$ Laboratoire d'Annecy-le-Vieux de Physique des Particules, CNRS/IN2P3, Annecy-le-Vieux, France
}

12 Astroparticule et Cosmologie (APC), CNRS, Université Paris VII, Paris; UMR 7164 (CNRS, Université Paris VII, CEA, Observatoire de Paris), France

13 Dublin Institute for Advanced Studies, Ireland

14 Landessternwarte, Universität Heidelberg, Königstuhl, Germany

15 Laboratoire de Physique Théorique et Astroparticules, CNRS/IN2P3, Université Montpellier II, Montpellier, France

16 Universität Erlangen-Nürnberg, Physikalisches Institut, Germany

17 Laboratoire d'Astrophysique de Grenoble, INSU/CNRS, Université Joseph Fourier, Grenoble, France

18 Institut für Astronomie und Astrophysik, Universität Tübingen, Germany

19 LPNHE, Universités Paris VI \& VII, CNRS/IN2P3, Paris, France

20 Institute of Particle and Nuclear Physics, Charles University, V Holesovickach 2, 18000 Prague 8, Czech Republic

21 Institut für Theoretische Physik, Lehrstuhl IV, Ruhr-Universität Bochum, Germany

22 University of Namibia, Windhoek, Namibia

23 Obserwatorium Astronomiczne, Uniwersytet Jagielloński, Kraków, Poland

24 Nicolaus Copernicus Astronomical Center, Warsaw, Poland

25 School of Physics \& Astronomy, University of Leeds, UK

26 School of Chemistry \& Physics, University of Adelaide, Australia

27 Toruń Centre for Astronomy, Nicolaus Copernicus University, Toruń, Poland

28 European Associated Laboratory for Gamma-Ray Astronomy, jointly supported by CNRS and MPG 